\title{
A Dynamic Invasive Weeds Optimization Applied to Null Control of Linear Antenna Arrays with Constrained DRR
}

\author{
Elhadi Kenane1, Haddi Bakhti', Miloud Bentoumi'1, and Farid Djahli2 \\ ${ }^{1}$ Department of Electronics, LGE Laboratory, University Mouhamed Boudiaf of M'sila, M'sila, 28000, Msila, Algeria \\ ${ }^{2}$ Department of Electrical Engineering, University of Setif1, Setif 1, 19000, Setif, Algeria \\ Corresponding author: E. Kenane (e-mail: elhadi.kenane@ univ-msila.dz).
}

\begin{abstract}
In the present work, a dynamic stochastic method is proposed and used for the synthesis of uniform linear antenna arrays. The proposed method combines the classical invasive weeds optimization (IWO) and the mutation process, which makes it robust, simple and shows flexibility to be adapted. The dynamic IWO applies the mutation process in the calculation of standard deviation during the spatial dispersal process of produced seeds while keeping the mean at the parent plants. In the mutation process, if special conditions were achieved, the standard deviation would be re-initialized. This proposed method tries to achieve an optimal array pattern by acting on the relative amplitude excitation of each element in the linear array for an optimal inter-element spacing. The optimal array pattern has deep or broad nulls in some directions of interferences with low sidelobes level. The objective of the synthesis is to get amplitude excitations with low dynamic range ratio (DRR), which facilitates the design of beamforming feed network. To illustrate the robustness of the proposed method, numerical examples are presented and compared with the obtained results using bees algorithm (BA), bacterial foraging algorithm (BFA), real genetic algorithm (RGA), and the corresponding reference array pattern for each example. ${ }^{1}$
\end{abstract}

INDEX TERMS Antenna array synthesis, dynamic invasive weeds optimization, nulls control, dynamic range ratio.

\section{INTRODUCTION}

$\mathbf{T}$ HE recent huge development in wireless communications needs permanent enhancements in antenna arrays characteristics to respond, efficiently, to the request of the dynamic environment. The most studied characteristics in the literature are the sidelobe level (SLL) suppression [1], the production of nulls in the directions of interferences [2], and the shaped power pattern [3]. In mobile communications, the linear antenna array topologies are very popular [4] because they offer the possibility to modify the inter-element spacing and the complex excitations (amplitudes and/or phases) of the array. In this work, we are interested in the latter possibility, which is the variation of complex amplitudes.

The synthesis problem of imposing deep and/or broad nulls in the direction of interference while the main-beam is oriented to the users is known as beamforming. Usually, this kind of synthesis problem has been done considering many constraints such as minimum SLL, a fixed first null beam width (FNBW), and a dynamic range ratio (DRR) less than a given threshold in the current distribution. Then, an additional cost function is used [5,6]. Another constraint presented in the mutual coupling, which can be avoided by using an interelement spacing equal to or greater than half a wavelength [7, 8].

Since the analytical synthesis techniques have many limitations such as being stuck in local minima, the evolutionary methods, inspired from natural phenomena, are increasingly employed to solve antenna synthesis problems [9]. Among the efficient and recent methods used to synthesize an antenna array, one can cite neural networks (NN) [10], real genetic algorithm (RGA) [11], bacterial foraging algorithm (BFA) [12], biogeography-based optimization (BBO) [13], bees colony algorithm (BA) [14], particle swarm optimization (PSO) [15], cuckoo search algorithm (CSA) [16], and jaya algorithm (JA) [17]. In [18], the authors proposed a new algorithm denoted IWO, which is a stochastic method inspired by the behavior of real colonizing weeds in nature. The antenna array synthesis using IWO became popular through many works in the literature [19].

${ }^{1}$ This work was supported by the Algerian Ministry of Higher Education and Scientific Research via funding through the PRFU project no. A25N01UN280120190001. 
Often, the IWO algorithm is used to solve real continuous problems; but in some cases, the binary coding is also applied [20]. To enhance the IWO algorithm, many researchers worked on its development in many hybrid versions. A hybrid IWO with iterative Fourier technique (IWO-IFT) has been developed to synthesize a large planar thinned array [21]. Other hybrids IWO were applied for synthesizing antenna arrays as in [22]-[24]. In the same way, a modified IWO is used to design a compact coplanar waveguide CPW-fed printed ultra-wideband antenna [25]. A modified IWO was also proposed for electricity price forecasting. It is based on reducing the standard deviation of a weed according to its cost function [26]. In addition, multi-objective invasive weed optimization (MOIWO) algorithm, based on the nondominated sorting of the solutions, has been used to optimize the pulse durations and the switch-on instants of the time-modulated elements of a conformal phased antenna array [27].

Previously, we proposed and used a modified IWO for the synthesis of both cosecant linear antenna array in [28] and non-uniform linear array in [29]. In this paper, the proposed dynamic IWO (DIWO) is used to synthesize linear antenna arrays to find optimal amplitude weights that allow achieving an array pattern imposing a broad null and suppressing the sidelobe levels with keeping the main beam width of a reference pattern. The DIWO results are compared with those of similar work using RGA [30], BFA [12], and BA [14]. Each example is compared to a uniformly excited equidistant array or Chebyshev array as in [12] and [14]. Four design examples of a linear array are presented, with respectively 12,16 , and 20 elements, in which the dynamic range ratio $(D R R)$ is taken into consideration. In the antenna array synthesis problem, it is important that $D R R$ of excitation amplitudes must be low to make the implementation of the array feeding feasible in practice [31].

\section{LINEAR ARRAY MODEL}

Consider a uniform linear array consisting of $2 N$ antenna elements as shown in Fig. 1. The array is assumed symmetric about its center. The Array Factor $(A F)$ is calculated based on

$$
A F(\theta)=2 \sum_{n=1}^{N} I_{n} \cos \left(k(n-0.5) d\left(\cos \theta-\cos \theta_{0}\right)\right)
$$

where

$k=2 \pi / \lambda$ and $\lambda$ is the wavelength, $N$ is the number of elements on each side of the array, $d$ is the inter-element spacing, $\theta$ is the observation angle from the axis of the array and $\theta_{0}$ is the pointing angle of the main beam (at broadside $\theta_{0}=90^{\circ}$ ). $I_{\mathrm{n}}$ denotes the current excitation of the $n^{\text {th }}$ element on each side of the array. Only the amplitude will be adjusted during the optimization process, the phase distribution is maintained constant and equal to zero.

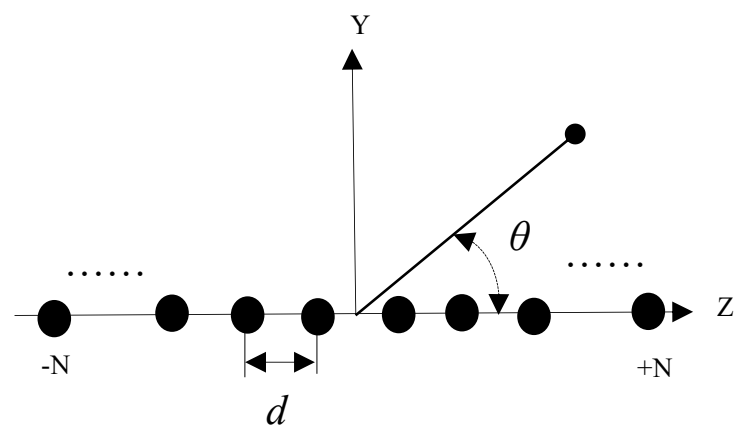

FIGURE 1. Linear array with $2 N$ isotropic elements positioned along the $z$ axis.

To examine the robustness of the proposed technique, two synthesis problems will be considered.

In the first part, the linear array synthesis will be done by considering two examples ( $2 N=12$ and 16 elements), which can provide an array pattern with two deep nulls, on each side of the main beam, with an acceptable level of the sidelobes. The nulls are imposed at the second and the third peaks of the reference pattern (uniform excitation $I_{\mathrm{n}}=1$ and inter-element spacing $d=\lambda / 2$ ). The expression (1) of $A F$ is used to obtain the amplitude excitations and the inter-element spacing $d$ that impose nulls towards specified directions and reduce the $S L L$, while keeping the beam-width of the main lobe equal to that of the reference pattern. These requirements are imposed using the following fitness function [30].

$$
\begin{aligned}
C F_{1}=C_{1} \frac{\mid \prod_{m=1}^{M} A F\left(\theta=n u l l_{m} \mid\right.}{\left|A F\left(\theta_{0}\right)\right|}+ & C_{2} \sum_{K=1}^{K} H(k)\left(Q_{k}-\delta\right)+ \\
& C_{3}\left(B W_{C}-B W_{d}\right)
\end{aligned}
$$

where

$M$ is the number of nulls on either side of the main beam. Generally, the number of nulls that can exist on the pattern of an array depends on many factors such as pattern geometry, number of elements, inter-element distance, and method of feeding. $A F\left(\theta_{0}\right)$ is the value of the array factor in the direction of the main beam. The second term of equation (2) is used to minimize the $S L L$ to a given threshold $\delta$. $k$ denotes the number of peaks of sidelobes in the reference pattern, $Q_{\mathrm{k}}$ is the sidelobe level (in $\mathrm{dB}$ ) achieved by the adjusted parameters ( $I_{\mathrm{n}}$ and $d$ ) at the $k^{\text {th }}$ peak, and $\delta$ is the desired threshold of the sidelobes level (in $\mathrm{dB}$ ). $H(k)$ is defined as

$$
H(k)=\left\{\begin{array}{l}
1,\left(Q_{k}-\delta\right)>0 \\
0,\left(Q_{k}-\delta\right) \leq 0
\end{array}\right.
$$

The third term is introduced to keep the bandwidth narrower than that of the reference pattern (uniform excitation with $d=\lambda / 2) . B W_{\mathrm{c}}$ and $B W_{\mathrm{d}}$ are, respectively, the calculated and the desired beam width. $\mathrm{C}_{1}, \mathrm{C}_{2}$, and $\mathrm{C}_{3}$ are the weight factors depending on the importance of each term. 
As in [30], we try to achieve an array pattern with deep nulls in some directions where the value of $\mathrm{C}_{1}$ must be higher than the values of $\mathrm{C}_{2}$ and $\mathrm{C}_{3}$. The value of the threshold $\delta$ is taken from the corresponding reference pattern $\left(I_{\mathrm{n}}=1, d=\lambda / 2\right)$ for the first two examples $(2 \mathrm{~N}=12,16)$. For each case, the threshold (desired SLL) $\delta$ is fixed to $-20 \mathrm{~dB}$ which is corresponding, approximately, to the level of the third peak in each example. For the examples of $2 \mathrm{~N}=20$, another function ESL will be used with a desired threshold $\delta=-28$ $\mathrm{dB}$, which is slightly above $30 \mathrm{~dB}$ of the reference pattern (30 dB Chebyshev array pattern).

The dynamic range ratio can be defined by the ratio between upper and lower value of amplitude excitations in an antenna array. An array pattern with low side lobe level can be easily achieved but with high DRR. A tradeoff between these two parameters must be taken into account. Minimizing the dynamic range ratio (DRR) is very useful to simplify the design of antenna array feed networks, which makes the implementation of the array feeding feasible in practice. Likewise, low DRR helps to reduce the effect of mutual coupling between array elements.

In some works, such as in [32], the DRR is reduced just by limiting the search interval of amplitude excitations. In this paper, to minimize the dynamic range ratio, another term can be introduced in the cost function; its expression is given by

$$
D R R=\frac{\max \left(I_{n}\right)}{\min \left(I_{n}\right)}
$$

In the second part of our study, we carry out a synthesis of a 20-element linear array $(2 N=20)$ by controlling the amplitude-only, which allows obtaining an array pattern with deep null in a given direction for the first example and a broad null in the desired direction for the last example. These goals can be achieved by minimizing the following cost function

$$
\begin{array}{r}
C F_{2}=\sum_{\theta=0}^{180^{0}}\left(W(\theta)\left|A F_{c a l}(\theta)-A F_{d}(\theta)\right|+E S L(\theta)\right)+ \\
D R R
\end{array}
$$

where

$A F_{\text {cal }}(\theta)$ stands for the achieved pattern using DIWO while $A F_{d}(\theta)$ denotes the desired pattern which can be given by

$$
A F_{d}(\theta)=\left\{\begin{array}{lr}
0, & \text { if } \theta=\theta_{i} \\
\text { initial patern, } & \text { elsewhere }
\end{array}\right.
$$

The term $W$ is used to control the null depth and position while $E S L$ controls the maximum peak sidelobe level. For a fair comparison, the values of the parameters $W$ and ESL are taken similar to those used in [14],

$$
W(\theta)=\left\{\begin{array}{l}
50, \text { if } \theta=\theta_{i} \\
1, \text { elsewhere }
\end{array}\right.
$$

and

$$
\operatorname{ESL}(\theta)=\left\{\begin{array}{c}
5, \text { if } M S L L>-28 d B \\
0, \quad \text { otherwise }
\end{array}\right.
$$

where MSLL represents the maximum sidelobe level and $\theta_{i}$ represents the direction of null.

\section{DYNAMIC IWO}

Modifications were made on the optimization algorithms to increase the speed of convergence and enhance the robustness of the classical algorithm. IWO is a new powerful method, compared to many other traditional metaheuristic methods. This method is based on the colonization phenomenon of real invasive weeds in agriculture [18]. The invasive weeds present a high resistivity and adaptability allowing them to occupy all the free space around cropping fields and grow to flower and generate new seeds. The optimization process starts from random parameters $\left(I_{\mathrm{n}}\right.$ and $d$ ) of a uniform linear array. These parameters will be optimized to place symmetric wide nulls on either side of the main beam. In the modified algorithms of IWO, many works try to find the model of spatial dispersal, which can provide good dispersion of seeds around the parent plant with improved performances. In our dynamic IWO, the model of spatial dispersal uses, iteratively, a mutation process in the calculation of standard deviation. The number of produced seeds depends on the fitness value of their parents. The produced weeds will grow randomly and become new plants in the colony. The algorithm of dynamic IWO proceeds as follows:

\section{A. INITIALIZATION}

Firstly, a population of plants will be generated, randomly, and spread over the search space. This initial population is denoted by $P O P=\left[P_{1}, P_{2}, \ldots, P_{\text {pop_ini }}\right]$ where $p o p \_i n i$ is the number of plants in the population. Each plant in the colony is treated as a proposed solution in the search space. Our problem has $(N+1)$ dimensions that can be represented by a vector $P_{\mathrm{i}}$ containing the excitation variables and the inter-element spacing. The vector $P_{\mathrm{i}}$ is termed as $P_{i}=\left[I_{1}, I_{2}, \ldots, I_{\mathrm{N}}, d\right]$.

\section{B. EVALUATION}

The evaluation of each plant is based on the associated fitness value which can give information about how good the plant is. To evaluate the plants, a cost function will be defined by the designer. The minimum and the maximum values of the cost function (fitness) correspond, respectively, to the best plant (solution) and the worst plant in the colony.

\section{REPRODUCTION}

According to the fitness value, each plant can produce a number of seeds varying from zero up to a maximum number. The better plant in the colony produces a maximum number of seeds while the worst plant does not produce any seed. The number of produced seeds for a given plant is calculated by 


$$
N_{s}\left(P_{i}\right)=\text { integer }\left[m s+\left(\frac{M_{S}-m s}{B C-W C}\right)\left(C\left(P_{i}\right)-W C\right)\right]
$$

where $M s$ and $m s$ are, respectively, the maximum number and the minimum number of seeds. While $B C, W C$, and $C\left(P_{i}\right)$ represent, respectively, the cost function (fitness) of the best plant, the cost function of the worst plant, and the cost function of the $i^{\text {th }}$ plant within the colony.

\section{SPATIAL DISPERSION}

Among the best characteristics of real weeds, we can cite their adaptation both for short-distance dispersal and long-distance dispersal [33]. Dispersal increases competition between parent and their offspring and decreases the chance of seed being damaged. In addition, dispersal reduces the probability of falling in a local minimum.

To simulate this ideal dispersion, the new seeds will be randomly dispersed around their parents, using a normal distribution with a zero mean and a standard deviation $S D$ that will be diminished iteratively as follows

$$
S D_{i t r}=\left(\frac{i t r_{\max }-i t r}{i t r_{\max }}\right)^{\bmod }\left(S D_{i n i}-S D_{f n l}\right)+S D_{f n l}
$$

where

itrmax $_{\text {max }}$ is the maximum number of iterations and itr is the actual iteration index. $S D_{i n i}$ and $S D_{f n l}$ are respectively the initial and final standard deviation values and mod represents the nonlinear modulation index, usually equal to 3 as suggested in [19]. In some works such as in [34], the value of mod is varied iteratively.

As depicted in Fig. 2, when the standard deviation dwindles iteratively, the search must be limited to the small areas around the reproducing plant, which has an accepted fitness level, which permits to increase the estimation accuracy [35].

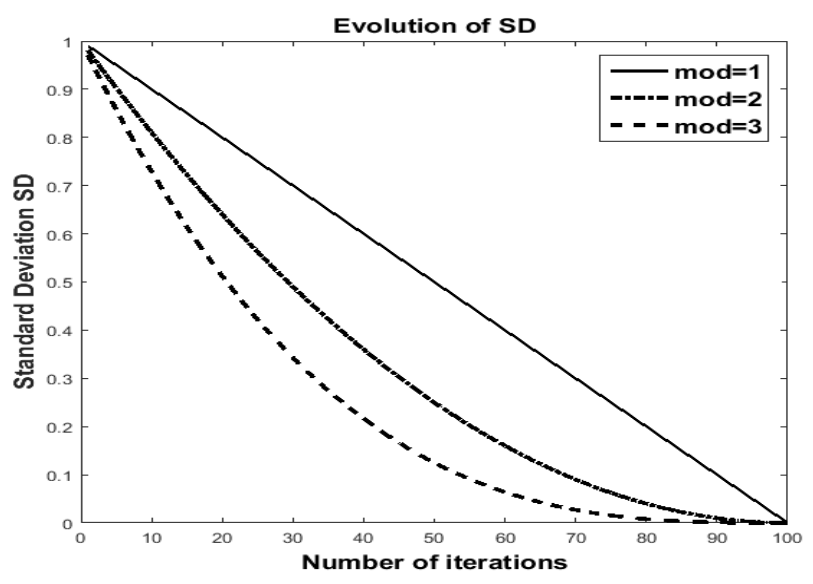

FIGURE 2. Evolution of classical IWO standard deviation for different mod values.

\section{E. MUTATION}

Unlike the conventional IWO, spatial dispersion procedure is modified using some other hybrid process. Many works of modified IWO demonstrate that the choice of standard deviation value is paramount in obtaining performance improvements such as fast convergence. Several studies highlight the significance of obtaining its optimal value. For instance, in [26], the produced seeds are selected randomly from the feasible solutions around their parent plants in a neighborhood with a normal distribution. In [27], for each plant, the value of the standard deviation is determined based on its ranking in the actual population, where the standard deviation will be varied periodically [35].

To enhance the explorative power of the classical IWO method, we incorporate the mutation process of the genetic algorithm to modify iteratively the standard deviation $S D_{\text {iter, }}$, which alter the distribution of new seeds around their parent plant. Classical genetic algorithm uses the mutation operation with a fixed probability rate, whose the inconvenient is that the best individual in the population will be selected several times. In our work, to avoid falling in local minima, a dynamic mutation probability is used. It consists in moving the search space during the optimization process to other promising search areas which can contain global minima, as illustrated in Fig. 3.

In our dynamic IWO, a mutation process of the GA will be used in the determination of the spatial dispersion parameters [36]. Initially, a probability $P_{\mathrm{m}}$ is given. If $P_{\mathrm{m}}$ is less than a random value in the interval $[0,1]$, the actual value of the standard deviation $S D_{\text {itr }}$ will be replaced by its initial value $S D_{\text {ini. }}$ Otherwise, the standard deviation $S D_{\text {itr }}$ will be calculated using (10).

The proposed algorithm is as follows:

Step 1: A random number is generated.

Step 2: A fixed probability of mutation $P_{m}$ is chosen.

Step 3: If the random number is greater than $P_{m}$ then

SDitr $=S D_{\text {ini }}$;

Else

$S D_{\text {itr }}$ is calculated from (10).

Step 4: Repeat this algorithm for each iteration.

The value of the mutation probability $P_{0}$ is chosen empirically. In our paper, this initial value is fixed to 0.8 , while the probability of mutation $P_{\mathrm{m}}$ will dwindle iteratively according to equation (11).

$$
P_{m}(i t r)=1-P_{0}^{\left(1-\frac{i t r}{n b r_{-} i t r}\right)}
$$

where $n b r_{-} i t r$ is the maximum number of iterations.

As $P_{\mathrm{m}}$ decreases iteratively, the standard deviation will be reduced when the cost function is near to its fitted value. According to equation (11), the dynamic nature of the proposed method allows the ratio of mutation operator to be changed linearly during the search progress. It starts from a 
ratio of $100 \%$ till it achieves a mutation ratio of $0 \%$ by the end of the search process $\left(P_{\mathrm{m}}=1\right.$ at the last iteration).

\section{F. LIMITATION}

The produced seeds will grow and will be added to the colony, where the population of plants grows fast and reaches its maximum number of plants pop_max. In this scenario, the population will be shrunken using a competitive exclusion. This process will keep, iteratively, only the best pop_max ${ }^{\text {th }}$ plants in the colony, while removing the rest.

\section{G. STOP CRITERION}

The simulation process continues until the maximum number of iterations is reached. The best plant in the final population will then be taken as an optimal solution.

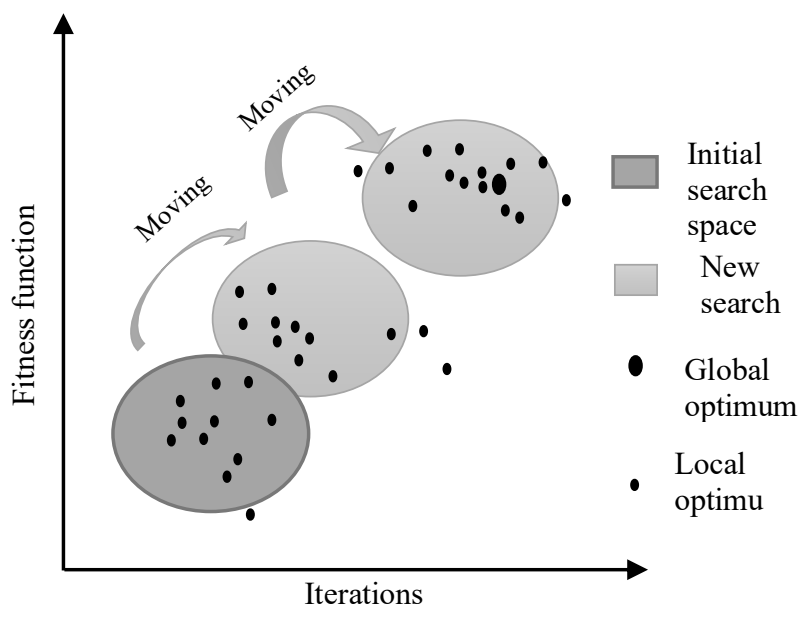

FIGURE 3. Dynamic IWO strategy

\section{NUMERICAL RESULTS}

The proposed dynamic IWO is applied to the synthesis of various linear array designs. The design goal is to impose nulls in the directions of interfering signals and suppress sidelobe levels, with respect to the main beam for a reference array, by controlling the amplitude excitation of each element array. Four design examples of uniform linear arrays are given here. The number of elements $(2 N)$ is, respectively, set to 12,16 , and 20. For all examples, the array is assumed to be symmetric about its center with an inter-element spacing $d$ and the scan angle of the main beam is fixed to $90^{\circ}$. In order to illustrate the performance of the proposed algorithm, the obtained results using our dynamic IWO (DIWO) method are compared to those of similar work using RGA [30] for the first two examples, while the last two examples are compared to those using BA and BFA algorithms. In our study, the $D R R$ is taken into consideration. For each example, both instantiations are compared to a reference pattern (uniformly excited array or Chebyshev array).
In each example, the size of the initial population, pop_ini, is set to $4 \mathrm{dim}$, where $\operatorname{dim}$ is the problem dimension. The maximum number of population $p_{0} p_{-} \max$ is twice the initial population pop_ini. For the standard deviation, SDini must be high to allow a good exploration of the search area (1 to 5 $\%$ of the range of the variable) as in [19], while the final SD, $S D f n l$, must be smaller than the precision criteria of the optimized variable. In the present study, $S D_{i n i}$ and $S D_{f n l}$ are fixed to 0.05 and 1e-7, respectively. The maximum number of iterations is fixed to 500 for the first part and 1000 for the second part.

In the first part of this study ( $2 N=12,16$ elements), DIWO is used to find deep nulls imposed at the locations of the second and third peaks of the reference pattern (uniformly excited array with $d=\lambda / 2$ ). The constraint is to reduce the SLLs while keeping the beam width close to that of the reference pattern. Here, the amplitude weights of the current distribution along the array are controlled with optimal inter-element spacing $d$. The amplitude weights can vary from zero to one, and $d$ can vary from $\lambda / 2$ to $\lambda$. The current distributions are assumed symmetric about the array center. Each plant in the population is considered as a vector of the amplitude excitations and the inter-element spacing of the array elements. The dimension of the problem becomes $(N+1)$, where the first $N$ values represent the amplitude weights, while the $(N+1)^{\text {th }}$ value represents the inter-element spacing. The obtained patterns, which use the control of amplitude weights and inter-element spacing, using DIWO for $2 N=12$ and 16 elements are presented in Fig. 4 and Fig. 5, respectively. As shown, these two figures present two deep nulls at the locations of the first and second peaks of the corresponding reference pattern. Likewise, Fig 4 and 5 present a considerable reduction in the sidelobes level, while maintaining some characteristics of the reference pattern such as beam width.

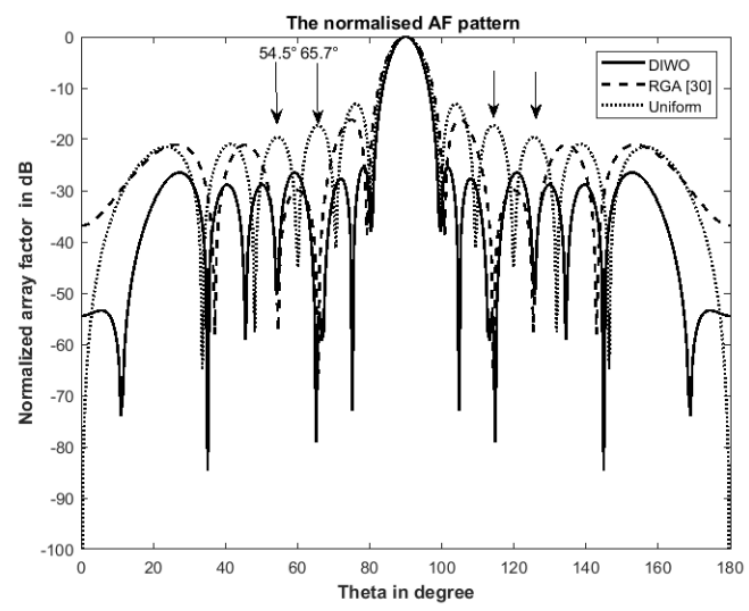

FIGURE 4. Comparison of normalized array factor with excitation coefficients by DIWO (solid line), RGA (dashed line), and uniform array (dotted line) for $2 N=12$, Nulls towards $54.5^{\circ}$ and $65.7^{\circ}$ 


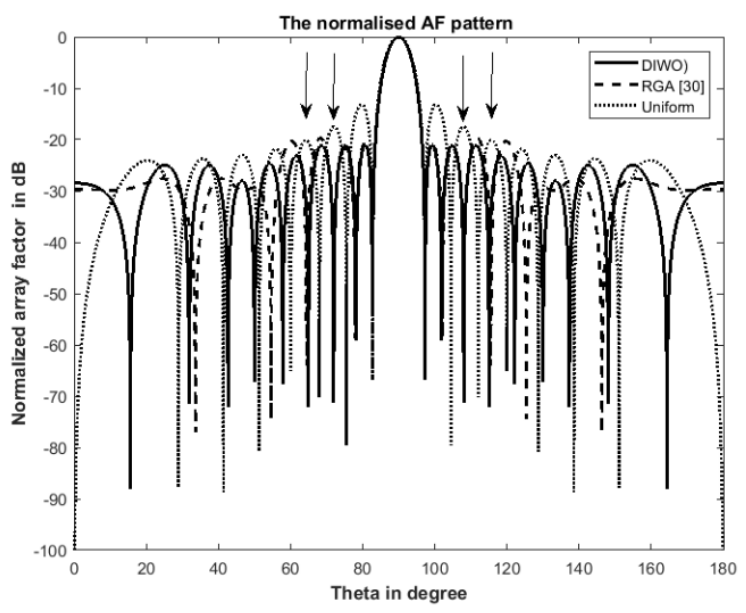

FIGURE 5. Comparison of normalized array factors with excitation coefficients by DIWO (solid line), RGA (dashed line) and uniform (dotted line) for $2 N=16$ with nulls imposed at the $2^{\text {nd }}\left(\theta=72^{\circ}\right)$ and $3^{\text {rd }}\left(\theta=64.4^{\circ}\right)$ peaks.

Table I presents all the results extracted from Fig. 4 and Fig. 5, while Table II indicates the optimal amplitude weights which are obtained using DIWO, with optimal inter-element spacing for each array configuration. For a comprehensive comparative evaluation, the obtained weights were contrasted with the best result of the other methods (RGA) with the same array configuration under equal simulation conditions and many runs. As it can be seen, the optimized array patterns, for $2 N=12$ and 16, present deep nulls, on each side of the main beam, at the second and third peaks. Also, Fig. 4 and 5, both illustrate a reduction in the maximum peak of the SLL (PSLL) with the obtained amplitude weights and the inter-element spacing compared to that of the corresponding reference pattern $\left(I_{\mathrm{n}}=1\right.$ and $\left.d=\lambda / 2\right)$. For each case, improved results are obtained with dynamic IWO. For $2 N=12$ elements, the first null at the location of the second peak $\left(\theta=65.7^{\circ}, 114.3^{\circ}\right)$ is deeper than $-69.5 \mathrm{~dB}$ where the second null imposed at the location of the third peak $\left(\theta=54.5^{\circ}, 125.5^{\circ}\right)$ is deeper than -100 $\mathrm{dB}$ with a dynamic range ratio equal to 3.6. The sidelobe level observed is $-22.6 \mathrm{~dB}$; it is reduced by more than $6 \mathrm{~dB}$ compared to that achieved by RGA $(-16.2 \mathrm{~dB})$. For the optimized array using RGA, the nulls at the locations of the second and third peaks have depths of, respectively, $-66.5 \mathrm{~dB}$ and $-82.8 \mathrm{~dB}$, with a $D R R$ equal to 6.6. The second example concerns an array of $2 N=16$ elements, the achieved $D R R$ is 1.7 for DIWO while it is 2.5 for RGA. The $S L L$ is reduced by more than $8 \mathrm{~dB}$ (from $-13.1 \mathrm{~dB}$ to $-21.1 \mathrm{~dB}$ ).

As it is shown in this example $(2 N=16)$, our goal is perfectly achieved by determining an optimal set of excitation currents of antenna elements. This optimal set has a lower DRR and help to obtain an array pattern with minimum SLL, and narrow beam width.
TABLE I. Pattern Properties of a Linear Array of $2 \mathrm{~N}=12$ and 16 Elements (Extracted from Fig. 4 and Fig. 5)

\begin{tabular}{|c|c|c|c|c|c|}
\hline & & & Uniform & RGA & DIWO \\
\hline \multirow{4}{*}{12} & \multirow{2}{*}{$\begin{array}{l}\text { Null depth } \\
\qquad(\mathrm{dB})\end{array}$} & $2^{\text {nd }}$ peak & -17.2 & -63.0 & -69.6 \\
\hline & & $3^{\text {rd }}$ peak & -19.6 & -85.1 & $<-100$ \\
\hline & FNBW in $\left(^{\circ}\right)$ & & 19.6 & 21.6 & 18.6 \\
\hline & PSLL (dB) & & -13.1 & -16.2 & -22.6 \\
\hline \multirow{4}{*}{16} & \multirow{2}{*}{$\begin{array}{l}\text { Null depth } \\
\text { (dB) }\end{array}$} & $2^{\text {nd }}$ peak & -17.5 & -83.9 & -72.2 \\
\hline & & $3^{\text {rd }}$ peak & -20.1 & -69.1 & -72.2 \\
\hline & $\mathrm{FNBW}$ in $\left(^{\circ}\right)$ & & 14.5 & 14.5 & 14.5 \\
\hline & PSLL (dB) & & -13.1 & -19.7 & -21.1 \\
\hline
\end{tabular}

TABLE II. Excitation Results for the Linear Array using the Dynamic IWO Method

\begin{tabular}{|c|c|c|c|}
\hline $\begin{array}{l}\text { Number } \\
\text { of } \\
\text { elements }\end{array}$ & Optima & $\begin{array}{l}\text { urrent excitation (normalized); } \\
d \text { in function of } \lambda\end{array}$ & $D R R$ \\
\hline \multirow[b]{2}{*}{12} & RGA & $\begin{array}{l}0.68760 .79690 .70230 .6753 \\
0.20920 .1201 ; 0.6262\end{array}$ & 6.6 \\
\hline & DIWO & $\begin{array}{l}0.76980 .65060 .59520 .4902 \\
0.29850 .2115 ; 0.7138\end{array}$ & 3.6 \\
\hline \multirow{2}{*}{16} & RGA & $\begin{array}{lllll}0.6049 & 0.5893 & 0.5405 & 0.5850 \\
0.3186 & 0.3795 & 0.4347 & 0.2377 ; \\
0.6006 & & & \end{array}$ & 2.5 \\
\hline & DIWO & $\begin{array}{llllll}0.9877 & 0.9911 & 0.8926 & 0.8395 \\
0.6699 & 0.5978 & 0.5858 & 0.5996 \\
0.5889 & & & \end{array}$ & 1.7 \\
\hline
\end{tabular}

In the second part, we consider a linear antenna array of $2 N=$ 20 elements with an inter-element spacing $d$ of one-half wavelength. In order to compare our method with the Bees Algorithm [14], the synthesis problem will be done by controlling only the amplitude. A wide null is placed at $30^{\circ}$ with $\Delta \theta=5^{\circ}$ for the first example and a deep null towards $14^{\circ}$ for the second one. In both examples, a Chebyshev pattern with $-30 \mathrm{~dB}$ sidelobe level is used as a reference pattern.

Array patterns are characterized by null depth, first null beamwidth $(F N B W)$, and $S L L$.

In the broad null example (synthesis of an array of $2 N=20$ elements for a desired wide null towards $30^{\circ}$ with $\Delta \theta=5^{\circ}$ ), we present (in Fig. 6) the radiation pattern obtained with DIWO, compared to BA, BFA, IWO/WDO patterns and the reference one. Pattern properties are tabulated in Table III while the corresponding excitation weights are reported in Table IV. For this example, the resulting pattern has a wide null imposed at $30^{\circ}$ with $\Delta \theta=5^{\circ}$. We note here that a null depth level, deeper than $-60 \mathrm{~dB}$, is achieved with a minimum $D R R$ (4.1), over the concerned region $\Delta \theta$, while the null depth level using BA is $-56.4 \mathrm{~dB}$. For IWO/WDO method [37], the resulted pattern has a wide deep null but it presents a DRR of 7.5 and a SLL of $-26 \mathrm{~dB}$. 


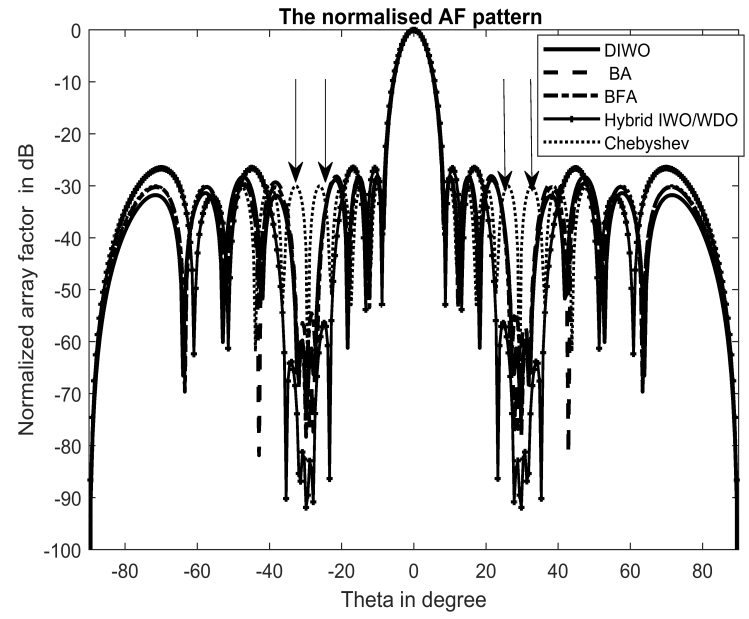

FIGURE 6. Comparison of normalized array factor with excitation coefficients by DIWO (solid), BA (dash-dotted), BFA (dashed), IWO/WDO (solid marked by + ), and Chebyshev (dotted) for $2 N=20$, Wide Null at $30^{\circ}$ with $\Delta \theta=5^{\circ}$.

TABLE III. Pattern Properties of an Array of $2 N=20$ Elements (Extracted from Fig. 6)

\begin{tabular}{cccc}
\hline \hline & Wide Null depth in $\mathrm{dB}$ & $\begin{array}{c}\text { FNBW in } \\
\text { degree }\end{array}$ & $\begin{array}{c}\text { PSLL in } \\
\mathrm{dB}\end{array}$ \\
\hline Chebyshev & - & 16.5 & -30 \\
\hline BA & -59.6 & 16.6 & -28.1 \\
\hline BFA & -54.5 & 16.6 & -28.2 \\
\hline $\begin{array}{c}\text { IWO/WDO } \\
{[37]}\end{array}$ & $>-74$ & 18.0 & 26.6 \\
\hline DIWO & -59.8 & 16.6 & -28.3 \\
\hline
\end{tabular}

TABLE IV. Current Excitation Weights for the Array of $2 \mathrm{~N}=20$ Elements with Wide Null Imposed at $30^{\circ}$

\begin{tabular}{|c|c|c|}
\hline Pattern & Optimal current excitation (normalized) & $D R R$ \\
\hline Chebyshev & 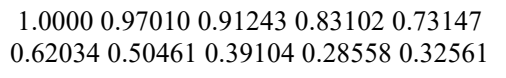 & 3.5 \\
\hline BA & $\begin{array}{rllll}1.0000 & 0.96710 & 0.94167 & 0.86812 & 0.69680 \\
0.56521 & 0.55146 & 0.46650 & 0.23989 & 0.25600 \\
\end{array}$ & 4.2 \\
\hline BFA & $\begin{array}{ccccc}1.0000 & 0.97404 & 0.93899 & 0.86503 & 0.70220 \\
0.56285 & 0.54207 & 0.46450 & 0.23882 & 0.25372\end{array}$ & 4.2 \\
\hline $\begin{array}{c}\text { IWO/WDO } \\
{[37]}\end{array}$ & 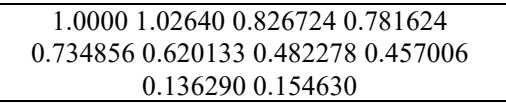 & 7.5 \\
\hline DIWO & $\begin{array}{rrrrr}0.9805 & 0.9765 & 0.9106 & 0.8444 & 0.7017 \\
0.5501 & 0.5469 & 0.4541 & 0.2367 & 0.2367\end{array}$ & 4.1 \\
\hline
\end{tabular}

In the last example, we synthesize an array of $2 N=20$ elements with the desired null at the peak of the second sidelobe of the Chebyshev pattern, which occurs at $94^{\circ}$. The obtained pattern is shown in Fig. 7. As it can be seen, the best design is obtained by DIWO, with a null depth around $-80 \mathrm{~dB}$.

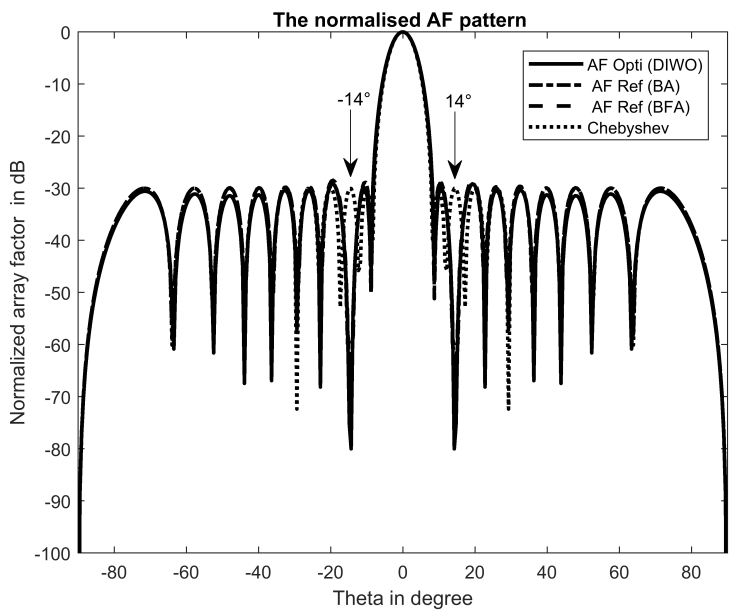

FIGURE 7. Comparison of normalized array factor with excitation coefficients by DIWO (solid), BA (dash-dotted), BFA (dashed), and Chebyshev (dotted) for $2 N=20$ Nulls towards $14^{\circ}$.

TABLE V. Current Excitation Weights for the Array of $2 N=20$ Elements with deep Null Imposed at $14^{\circ}$

\begin{tabular}{|c|c|c|}
\hline Pattern & Optimal current excitation (normalized) & $D R R$ \\
\hline Chebyshev & 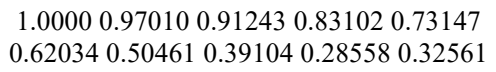 & 3.5 \\
\hline BA & $\begin{array}{ccccc}1.0000 & 0.98927 & 0.95488 & 0.88706 & 0.77968 \\
0.64053 & 0.48971 & 0.35057 & 0.24459 & 0.31066\end{array}$ & 4.1 \\
\hline BFA & $\begin{array}{ccccc}1.0000 & 0.99452 & 0.96393 & 0.89263 & 0.78084 \\
0.63995 & 0.48871 & 0.35188 & 0.24809 & 0.31423\end{array}$ & 4.0 \\
\hline DIWO & $\begin{array}{lllll}0.9693 & 0.9610 & 0.9257 & 0.8528 & 0.7427 \\
0.6093 & 0.4534 & 0.3312 & 0.2335 & 0.2752\end{array}$ & 4.2 \\
\hline
\end{tabular}

In Table V, we present a comparison between our results and results obtained using different stochastic methods, for a uniform linear array of 20 elements. These results concerns the optimal amplitudes excitation obtained by our dynamic IWO and those achieved by BA and BFA methods, for the same topology design. The comparison shows that our method allows to obtain better results in terms of reduced DRR (4.2), deep nulls and minimum SLL.

The results depicted in Fig. 4, 5, 6, and 7 illustrate the robustness of the proposed algorithm for the antenna array synthesis with deep nulls towards interference directions. To complete the study, the evolution of the minimum cost function (in $\mathrm{dB}$ ) in the colony is presented versus the number of iterations in Fig. 8. The evolution of standard deviation versus iterations using DIWO is presented in Fig. 9, where more than 94 mutations are shown. Fig. 10 illustrates the optimal distribution of excitation that gives the optimized array factor in Fig. 7, this distribution presents a DRR of 4.2. 


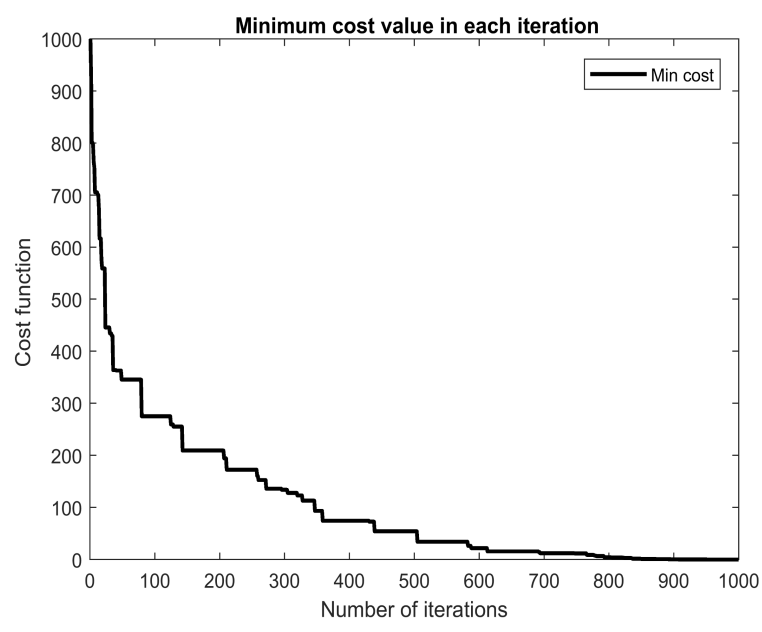

FIGURE 8. Convergence curve of the minimum cost function of the proposed method for $2 \mathrm{~N}=20 \mathrm{Null}$ at $14^{\circ}$.

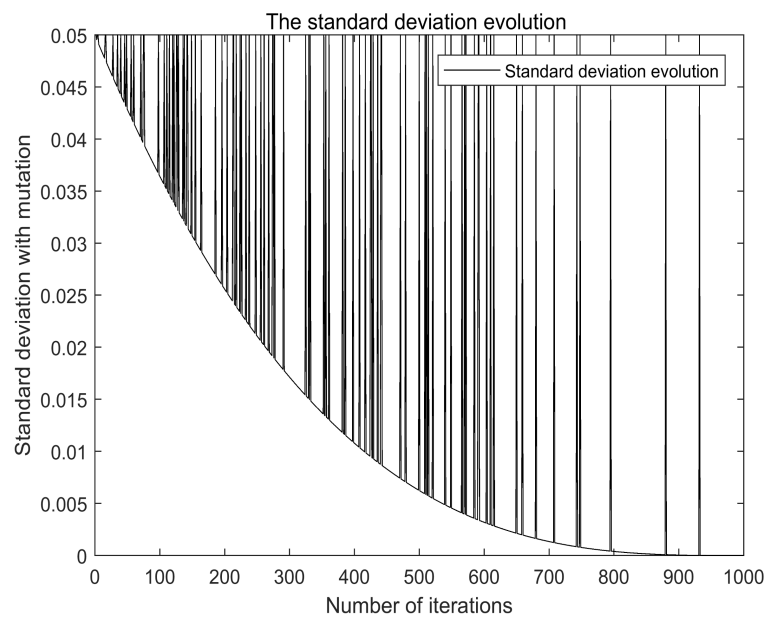

FIGURE 9. Evolution of standard deviation versus the number of iterations for the example of $2 \mathrm{~N}=20$ and null at $14^{\circ}$.

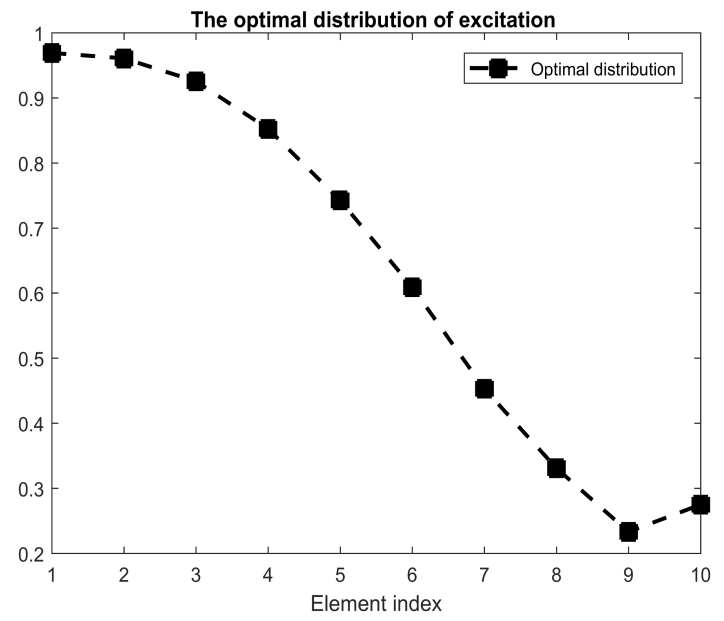

FIGURE 10. Optimal excitation distribution of linear array for the example cited in Fig. $7\left(2 \mathrm{~N}=20\right.$ and null imposed at $\left.14^{\circ}\right)$.

\section{CONCLUSION}

In this paper, a dynamic IWO was presented and successfully applied to the synthesis of the uniform linear antenna array. This synthesis problem was used to find the current excitation for each element in the array while leaving the phase unmodified. The DDR was also taken into account. The results showed that the dynamic IWO method gives normalized array factors with imposed nulls (deep or wide) in the desired directions and also with minimum side-lobe levels with the constraint of fixed main beam width and low DRR. The obtained patterns were compared with data from reference arrays and with BA and BFA or RGA optimized arrays, which illustrate the robustness of the proposed method, constrained on DRR, for placing single or multiple nulls (deep or broad). Moreover, the mutation process was used in the determination of the spatial dispersion $(S D)$ value, which allowed a good exploration of the search space and escaping from falling in local minima and helping maintain diversity in the colony. In consequence, the convergence has been accelerated. We plan for our future works to exploit our dynamic IWO for adaptive beamforming applications.

\section{REFERENCES}

[1] A. Das, D. Mandal, S. P. Ghoshal, and R. Kar, "Concentric circular antenna array synthesis for sidelobe suppression using moth flame optimization," International Journal of Electronics and Communications, vol. 86, pp. 177-184, 2018.

[2] S. U. Rahman, Q. Cao, M. M. Ahmed, and H. Khalil, "Analysis of Linear Antenna Array for minimum Side Lobe Level, Half Power Beamwidth, and Nulls control using PSO," Journal of Microwaves, Optoelectronics and Electromagnetic Applications, vol. 16, no. 2, pp. 577-591, 2017.

[3] J. Bai, Y. Liu, J. Cheng, P. You, and Q. Liu, "Shaped power pattern antenna array synthesis with reduction of dynamic range ratio," Progress in Electromagnetic Research Symposium (PIERS), Aug 2016, China, pp. 2444-2447, 2016

[4] C. Godara, "Applications of Antenna Arrays to Mobile Communications, Part I: Performance Improvement, Feasibility, and System Considerations," Proceedings of the IEEE, vol. 85, no. 7, pp. 1031-1060, 1997.

[5] M. Comisso, and R. Vescovo, "3D Power Synthesis with Reduction of Near-Field and Dynamic Range Ratio for Conformal Antenna Arrays," IEEE Transactions on Antennas and Propagation, vol. 59, no. 4, pp. 1164-1174, 2011.

[6] R. Vescovo, "Array Pattern Synthesis with Near-field Constraints, Reconfigurability by Phase-only Control and Reduction of Dynamic Range Ratio," IEEE Antenna and Propagation Society International Symposium, 1B, pp.774-777. 2005.

[7] D. M. Pozar, "The Active Element Pattern," IEEE Transactions on Antennas and Propagation, vol. 42, no. 8, pp. 1176-1178, 1994.

[8] C. A. Balanis, "Modern Antenna Handbook: part III Arrays and synthesis methods," John Wiley \& Sons, USA, 2008.

[9] S. K. Goudos, C. Kalialakis, and R. Mittra, "Evolutionary Algorithms Applied to Antennas and Propagation: A Review of State of the Art," International Journal of Antennas and Propagation, vol. 2016, pp. 1$12,2016$.

[10] L. Merad, F.T. Bendimerad, S.M. Meriah, and S. A. Djennas, "Neural Networks for Synthesis and Optimization of Antenna Arrays," Radio Engineering, vol. 16, no. 1, pp. 23-30. 2007

[11] L. Bian, "Low-side-lobe pattern synthesis of antenna array by genetic algorithm," Journal of Computer Applications, vol. 28, no. 7, pp. $1656-1658,2008$.

[12] K. Guney, and S. Basbug, "Interference Suppression of Linear Antenna Arrays by Amplitude-Only Control Using a Bacterial 
Foraging Algorithm," Progress in Electromagnetics Research, PIER, vol. 79, pp. 475-497, 2008.

[13] A. Sharaqa, and N. Dib, "Design of Linear and Elliptical Antenna Arrays Using Biogeography Based Optimization,” Arabian Journal for Science and Engineering, vol. 39, no. 4, pp. 2929-2939, 2014.

[14] K. Guney, and M. Onay, "Amplitude-Only Pattern Nulling of Linear Antenna Arrays with the use of Bees Algorithm," Progress in Electromagnetics Research, PIER, vol. 70, pp. 21-36, 2007.

[15] M. Khodier, and C. G. Christodoulou, "Linear Array geometry synthesis with minimum sidelobe level and null control using Particle Swarm Optimization," IEEE transaction on antennas and propagation, vol. 53, no. 8, pp. 2674-2679, 2005.

[16] H. Rakhshani and A. Rahati, "Snap-drift cuckoo search: A novel cuckoo search optimization algorithm," Applied Soft Computing, vol. 52, pp. 771-794, 2017.

[17] L. R. Jaya and N. Mary, "Synthesis of linear antenna arrays using Jaya, self-adaptive Jaya, and chaotic Jaya algorithms," International Journal of Electronics and Communications, vol. 92, pp. 54-63, 2018.

[18] A. R. Mehrabian, and C. Lucas, "A Novel Numerical Optimization Algorithm Inspired from Weed Colonization," Ecology Information, vol. 1, no. 4, pp. 355-366, 2006

[19] S. Karimkashi, and A. Kishk, "Invasive Weed Optimization and its Features in Electromagnetics," IEEE Transactions on Antenna and Propagation, vol. 58, no. 4, pp. 1269-1278, 2010.

[20] C. Veenhuis, "Binary Invasive Weed Optimization," Second World Congress on Nature and Biologically Inspired Computing, IEEE, Japan, pp. 449-454, 2010.

[21] X. Wang, Y. Jiao, Y. Liu, and Y. Tan, "Synthesis of Large Planar Thinned Arrays Using IWO-IFT Algorithm," Progress In Electromagnetics Research, vol. 136, pp. 29-42, 2013.

[22] Y. Y. Bai, S. Xiao, C. Liu, and B. Z. Wang, "A Hybrid IWO/PSO Algorithm for Pattern Synthesis of Conformal Phased Arrays," IEEE Transactions on Antennas and Propagation, vol. 61, no. 4, pp. 23282332, 2013.

[23] A. Dastranj, H. Abiri, and A. R. Mallahzadeh, "Two-dimensional synthesis and optimization of a broadband shaped beam reflector antenna using IWO and PSO algorithms," Int. J. RF Microwave CAE, vol. 25 , no. 2 , pp. $129-140,2015$

[24] Z. D. Zaharis, C. Skeberis, T.D. Xenos, P.I. Lazaridis, and J. Cosmas, "Design of a Novel Antenna Array Beamformer Using Neural Networks Trained by Modified Adaptive Dispersion Invasive Weed Optimization Based Data," IEEE Transactions on Broadcasting, vol. 59, no. 3, pp. 455-460, 2013.

[25] A. Dastranj, "Optimization of a Printed UWB Antenna: Application of the invasive weed optimization algorithm in antenna design," IEEE Antennas and Propagation Magazine, vol. 59, no. 1, pp. 48-57, 2017.

[26] R. Giri, A. Chowdhury, A. Ghosh, B. K. Panigrahi, and A. Mohapatra, "Electricity Price Forecasting A Hybrid Wavelet Transform and Evolutionary- ANN Approach," International Conference on Power Electronics, Drives and Energy Systems \& Power India 2010, pp. 1-6, 2010.

[27] W. T. Li, Y. Q. Hei, and X. W. Shi, "Synthesis of Conformal Phased Antenna Arrays With a Novel Multiobjective Invasive Weed Optimization Algorithm," Frequenz, vol. 72, no. 5-6, pp. 209-219, Apr. 2018

[28] E. H. Kenane, F. Djahli, and A. Bartil, "Synthesis of Cosecant Linear Antenna Array Pattern using a Novel Modified Invasive Weeds Optimization," Elektronika ir Elektrotechnika, vol. 21, no. 5, pp. 8689,2015

[29] E. H. Kenane, and F. Djahli, "Optimum design of non-uniform symmetrical linear antenna arrays using a novel modified invasive weeds optimization," Archives of Electrical Engineering, vol. 65, no. 1 , pp. 5-18, 2016

[30] B. Goswami, and D. Mandal, "A Genetic Algorithm for the Level Control of Nulls and Side lobes in Linear Antenna Arrays," Journal of King Saud University, Computer, and Information Sciences, vol. 25, no. 2, pp. 117-126, 2013.

[31] T. Lo, J. Wang, and J. Litva, "An Optimization Approach to Pattern Synthesis in the Design of Microstrip Phased Array Antennas," Digest on Antennas and Propagation Society International Symposium, IEEE, pp. 886-889. 1989.
[32] Z. Xu, Y. Liu, M. Li, and Y. Li, "Linearly Polarized Shaped Power Pattern Synthesis With Dynamic Range Ratio Control for Arbitrary Antenna Arrays," IEEE Access, vol. 7, pp. 53621-53628, 2019.

[33] S. R. Radosevich, J. S. Holt, and C. M. Ghersa, "Ecology of weeds and invasive plants: relationship to agriculture and natural resource management," John Wiley \& Sons, Ed. New Jersey, 2007.

[34] A. Basak, S. Pal, S. Das, and A. Abraham, "Circular Antenna Array Synthesis with a Differential Invasive Weed Optimization Algorithm," Tenth International Conference on Hybrid Intelligent Systems, IEEE Conference, USA, pp. 153-158. 2010.

[35] G. G. Roy, S. Das, P. Chakraborty, and P. N. Suganthan, "Design of Non-uniform Circular Antenna Arrays Using a Modified Invasive Weed Optimization Algorithm," IEEE Transactions on Antennas \& Propagation, vol. 59, no. 1, pp. 110-118, 2011.

[36] G. R. Hardel, D. Mandal, S. P. Ghoshal, and R. Kar, "Minimization of Side Lobe of Optimized Uniformly Spaced and Non-uniform Excited Time-Modulated Linear Antenna Arrays Using Genetic Algorithm," Lecture Notes in Computer Science, Swarm, Evolutionary, and Memetic Computing, Springer, vol. 7677, pp. 451$458,2012$.

[37] S. K. Mahto and A. Choubey, "A novel hybrid IWO/WDO algorithm for nulling pattern synthesis of uniformly spaced linear and nonuniform circular array antenna,” Int. J. Electron. Commun. (AEÜ), vol. 70 , no. 6 , pp. $750-756,2016$. 